\title{
LAS POLÍTICAS SOCIALES \\ EN EL WELFARE MIX
}

\section{Manuel Herrera Gómez}

Universidad de Granada

\begin{abstract}
RESUMEN
En Europa, los sistemas de protección social han entrado en crisis con el tránsito de la sociedad industrial a la postindustrial. Aún es un problema comprender en qué consiste este proceso "societario», proceso que comporta una redefinición de la seguridad social en el marco de un sistema de protección social más amplio. Las dificultades para acceder a nuevos sistemas de protección social están ligadas al siguiente hecho: prevalecen las principales instituciones - y sus correspondientes mecanismos- de seguridad social tipo industrial, es decir, una seguridad social diseñada sobre relaciones industriales y sobre problemas del mercado de trabajo; mientras que la seguridad social sólo puede funcionar bien si cuenta con un sistema más amplio, articulado e integrado de intervenciones de protección social. Dicho en otros términos, con una concepción global del welfare mix.
\end{abstract}

\section{EL BIENESTAR (WELL-BEING) MÁS ALLÁ DEL ESTADO DE BIENESTAR DE LA MODERNIDAD}

Ha llegado el momento de preguntarse cuál es el presente y el futuro de las políticas sociales en la sociedad europea, una sociedad que está modificando

${ }_{1}$ Este artículo forma parte de los resultados del proyecto PB98-1633, financiado por el Ministerio de Ciencia y Tecnología. Agradezco al profesor Julio Iglesias de Ussel (Universidad de Granada) las orientaciones y sugerencias que me ha hecho en la elaboración de este trabajo. 
los ejes del Estado de Bienestar. Dicho en otros términos, es necesario comprender el escenario que se dibuja en el horizonte para tener una representación sobre cómo cambian el sentido y las modalidades de practicar el trabajo social en todos los sectores y actividades.

Para afrontar este tema no nos detendremos en los recientes cambios del Estado Social ${ }^{2}$, aunque sí aludiremos a ciertos aspectos de su crisis para aclarar nuestras argumentaciones. Nuestro objetivo es ilustrar la siguiente perspectiva: el Estado de Bienestar ha sido el producto de la modernidad; en concreto, de una modernidad que ha confiado al Estado el objetivo de producir el bienestar social. La crisis de esta configuración anuncia el tránsito a una nueva sociedad que denominamos postmoderna. En ella, el bienestar (como well-being) se convierte en una tarea de la sociedad civil. Una función que es interna a un particular sistema relacional que conecta las diversas dimensiones del bienestar en el interior de las esferas civiles, y entre éstas y el sistema político-administrativo.

El punto de partida de estas páginas es el siguiente: la crisis de los sistemas de protección social construidos tras la II Guerra Mundial no significa la renuncia a formas más avanzadas y completas de garantías para los ciudadanos -que se extienden bajo ciertas condiciones a los inmigrantes residentes (denizens) y no residentes-. Más bien significa la crisis de cierto modelo de Estado de Bienestar - el neocorporativo-. Se abre una nueva fase histórica en la que es preciso reflexionar sobre el sentido de la protección social y cómo realizarla. Esta fase histórica contiene en su núcleo la idea y las prácticas de welfare mix. ¿Qué significa esta expresión? Éste es nuestro objetivo.

$\mathrm{Si}$, en la actualidad, todos los Estados europeos activan políticas de welfare mix, ¿qué cambia en el sentido y en las modalidades de realización de las políticas sociales? Nuestra tesis es que nos encontramos ante dos grandes modelos de welfare mix alternativos: el liberal/laboralista y el societario (o relacional).

Escoger una u otra vía implica profundas diferencias en el modo de entender y practicar el trabajo social. Trataremos de trazar estas diferencias desde la observación de la diversa posición "política» que el trabajo social asume en tales configuraciones. El análisis conduce a un interrogante muy estimulante: ¿las políticas sociales son y serán «políticas» en los próximos años? Nuestra tesis es que el sentido político del trabajo social debe asumir una nueva definición postmoderna. Se trata de identificar los principios-guía de revisión del «sentido político» de las políticas de welfare mix.

2 Véanse, entre otros, I. Colozzi (1987), M. Bulmer (1989), N. Barry (1990), C. Jones (1993), R. Montoro (1997), P. Donati (1998) y M. Herrera (1998a y 1998b). 


\section{II. ¿QUÉ ES EL WELFARE MIX?}

Para comprender qué es y cómo se configura el welfare mix es necesario tomar como punto de partida la crisis del modelo neocorporativo democrático del Estado de Bienestar y de su política social ${ }^{3}$.

El modelo neocorporativo, dominante en Europa hasta hace poco tiempo y presente aún en muchos Estados, se basa en una concepción de la ciudadanía social cuyos ejes fundamentales son los siguientes ${ }^{4}$ :

a) La ciudadanía es concebida como complejo de derechos-deberes referentes al individuo, si y cuando dicho individuo pertenece al Estado. La ciudadanía - con todos los derechos sociales correspondientes - es definida a partir del eje individuo-Estado, sin ningún tipo de mediaciones.

b) El gobierno de la sociedad, y por tanto de la gestión de la ciudadanía, tiene lugar mediante el compromiso entre el Estado y el mercado, en cuyo marco se resuelven los problemas de bienestar como compensación por déficit sobre el mercado.

c) Las políticas sociales son, en consecuencia, expresión del concierto entre los actores del Estado y los actores del mercado.

La crisis del modelo neocorporativo, acentuada a partir de los años ochenta, conlleva la emergencia de una concepción de la ciudadanía que se basa en:

a) La ciudadanía ya no es definida como pertenencia del individuo al Estado, sino como complejo de derechos-deberes de los «sujetos de ciudadanía», ya sean individuos o actores colectivos. La ciudadanía se presenta como la dimensión política de las relaciones originarias entre asociados y, por tanto, se entiende como derecho subjetivo de las personas y de las formaciones sociales intermedias en las que los individuos desarrollan su existencia.

b) El gobierno de la sociedad, y por tanto la gestión de la ciudadanía, se ubica en el cuadro de una concertación entre el Estado y una pluralidad de actores sociales, bien sean de mercado o no. Dicho brevemente, los actores del mercado ya no son los interlocutores privilegiados; a su lado, y a menudo en su puesto, están las «asociaciones» del privado social'.

c) En consecuencia, las políticas sociales se presentan como la expresión de una redistribución del poder y de las iniciativas entre un número mayor y cualitativamente diverso de actores de la sociedad. Entre ellos están los estatales y los procedentes del mercado, pero ya no ocupan una posición central o de relevancia sobre el resto de actores.

\footnotetext{
Véanse H. Wilensky (1976) y H. Wilensky y otros (1985).

Véase T. H. Marshall (1950).

Sobre el concepto de "privado social», véase P. Donati (1978).
} 
En la medida en que se afianza este nuevo escenario, el de la sociedad postindustrial, el Estado de Bienestar deja espacio a una sociedad del bienestar que se caracteriza por: la pluralización de los actores y la pluralización de los instrumentos de protección social. En tal configuración:

- Las políticas sociales ya no coinciden con las políticas públicas; dicho brevemente, la protección social ya no coincide con las instituciones estatales (del Estado-nación); la política social se presenta como una función social difusa.

- En cuanto que las sociedades postmodernas son sociedades altamente desnormativizadas, es inevitable que también los sistemas de protección social estén desreglados (de-regulation); en apariencia puede parecer una contradicción, pero no lo es: desregulación significa que los esquemas universales, uniformes y basados en normas-programas de tipo condicional dejan paso a esquemas selectivos, diversificados y orientados a objetivos.

Tanto la cultura como las estructuras organizativas de bienestar maduran la idea de que éste (well-being) debe ser expresión de la sociedad más que del Estado; y que los destinatarios de los programas deben jugar un rol más activo e incluso empresarial. Se abre camino la idea de los pro-sumers, es decir, de los ciudadanos como sujetos que son al mismo tiempo productores, distribuidores y consumidores de bienes y servicios de bienestar y, en general, de protección social.

En este marco se habla de welfare mix. Nuestra tesis es que el welfare mix, especialmente en España, es un proceso en acto que busca un nuevo paradigma, claro y consolidado, de políticas sociales. La mayor parte de los trabajadores sociales tiene una visión parcial. En general, lo entienden como fragmentación de las políticas sociales, o bien como un modo de superar la crisis del bienestar público (estatal) mediante «inyecciones» de bienestar privado, bien mercantil, bien de privado social. Sin embargo, el welfare mix conlleva innovaciones que orientan hacia un nuevo paradigma sociológico. Se trata de un paradigma relacional y morfogenético que está sometido a tendencias en acto, y que comporta nuevas metodologías de intervención que aún están en vía de experimentación.

La idea de welfare mix nace del siguiente hecho: en los años ochenta, con la crisis del binomio Estado-mercado en régimen neocorporativo, los sistemas societarios han debido recurrir a las familias y a las organizaciones (voluntariado, asociaciones, cooperativas sociales, fundaciones) de tercer sector. Sin embargo, este proceso no se detiene aquí. Una vez activado, la emergencia del tercer sector ha conllevado una serie de dinámicas más complejas.

Al principio del proceso, la expresión welfare mix significaba que el bienestar no podía asegurarse sólo por el Estado, ni tampoco por una concertación neocorporativa entre el Estado y los actores del mercado. Se debía recurrir a 
otros sujetos (privado social, tercer sector, familias y redes informales). De esta forma, el bienestar se presentaba como el producto y la expresión de una pluralidad de actores. Esa fase ilumina, en sentido empírico, los cuatro tipos de actores o instituciones que deben producir el bienestar: mercado, Estado, tercer sector y redes informales. Y lo deben hacer conjuntamente, sin dar prioridad absoluta a ninguno de ellos. Más bien deben actuar mediante relaciones de diferenciación e integración social.

A continuación, ya que estos actores tienen concepciones teóricas (códigos culturales) y prácticas sociales (sistemas de acción) diferentes en el modo de perseguir el bienestar, ha sido necesario aplicar a cada uno un diverso esquema analítico de significación del bienestar ${ }^{6}$. Esta fase ha servido para aclarar que, en sentido analítico, las cuatro dimensiones de los códigos culturales son: sus medios (económicos y técnicos), sus objetivos, sus normas sociales y sus valores últimos.

De esta forma, se han generalizado el concepto de bienestar y las prácticas para conseguirlo. Hoy no lo podemos entender sólo en términos materiales (bienestar como welfare), sino que debemos incluir también los componentes psicoculturales y relacionales (bienestar como well-being), que no son añadidos o secundarios, sino constitutivos y primarios del mismo bienestar material. Por tanto, el bienestar debe contemplarse como el producto de cuatro tipos de actores y de cuatro códigos culturales correspondientes, en relaciones complejas entre sí $^{7}$.

La generalización del concepto y su difusión se han trasladado a una fase lógica y temporal sucesiva. En ella, el mix que genera bienestar ha adquirido las características de un "fenómeno emergente» (en sentido fuerte y técnico). Como punto de llegada, se considera que hoy el concepto de welfare mix expresa la idea de que el bienestar es el producto y la expresión de una lógica compleja que es:

- Combinatoria en cuanto que reúne los sujetos de cuatro sectores y los elementos de sus códigos culturales.

- Reticular ya que debe producir bienestar apelando a las relaciones «internas» $\mathrm{y}$ "entre» (within y between) estos sectores, sus sujetos y sus códigos culturales.

- Emergente porque el bienestar se convierte en el resultado no predecible a priori de una autonomía de las interacciones e interdependencias creadas mediante procesos que adquieren mayor flexibilidad, competencia y, en general, una elevada movilidad y dinamicidad; ahora el bienestar debe observarse y perseguirse como "efecto emergente» (en sentido técnico, es decir, como producto de elementos que, al fundirse, originan una «composición» cuyas propiedades no derivan de los singulares componentes).

6 Véase P. Donati (1999a).

7 Véase P. Donati (1999b). 
Quien permanece anclado en el viejo orden normativo observa que las relaciones entre estos actores no responden a un cuadro claro y coordinado, que pueda "gobernarse» en términos de eficacia, eficiencia y equidad "relacional». La mayor parte de los analistas del Estado de Bienestar asumen posiciones defensivas y ven los cambios como una amenaza a las garantías sociales conquistadas en el pasado. Obviamente, hay razones válidas para sostener este punto de vista. Sin embargo, tal lectura de los fenómenos posee notables carencias para interpretar los procesos en acto y las perspectivas operativas sobre el futuro.

El problema es: ¿cómo entender y regular el nuevo orden de los servicios de bienestar (sin que perdamos calidad, eficacia, eficiencia y equidad)?

Antes de responder a este interrogante, es necesario dotarse de una representación adecuada de la sociedad postindustrial como sistema articulado en esferas diferenciadas entre sí que precisan integración mediante sus intercambios ${ }^{8}$. La representación puede realizarse mediante un diagrama de flujos, construido sobre cuatro sectores (esferas o ámbitos) distintos y sus respectivos actores (fig. 1).

\section{FIGURA 1}

Ambitos y actores de welfare mix

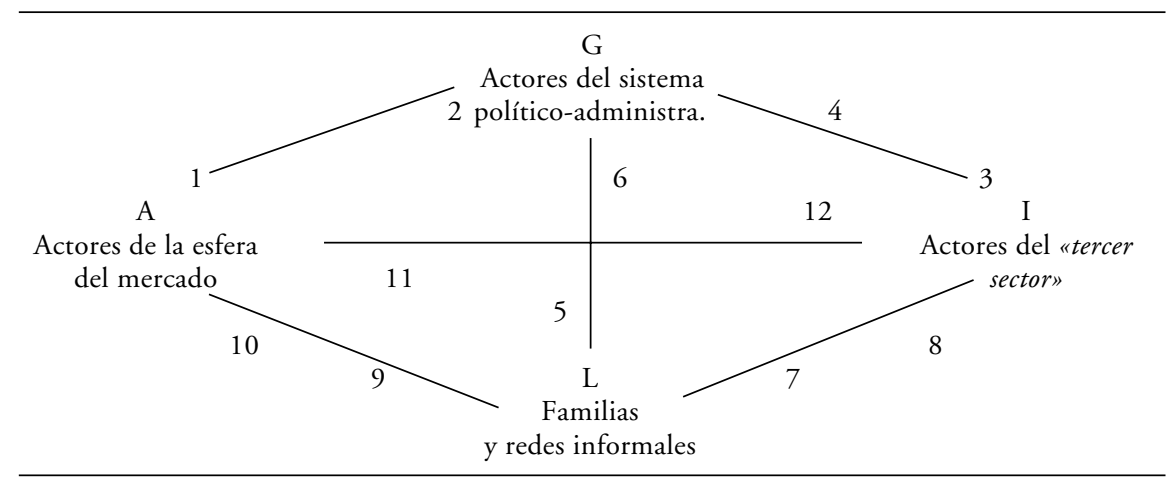

A) En la esfera del mercado trabajan las organizaciones sindicales, empresariales y de categoría.

G) En la esfera de la política encontramos actores que actúan por referencia al Estado: es la seguridad social garantizada o condicionada por el Estado.

I) En la esfera de la economía social (o mercado social) están los actores que solemos llamar de privado social o «tercer sector».

${ }^{8}$ Utilizo el esquema sociológico AGIL, inicialmente planteado por T. Parsons y reelaborado en clave relacional por P. Donati (1991: cap. 4). 
L) En la esfera de las comunidades primarias encontramos las familias y las redes informales de vida cotidiana (tagesmütter, "buen vecino", grupos de self help y mutual help, etc.).

La visión que sugerimos nos permite realizar una amplia proyección histórica. En la primera modernidad, el binomio Estado-Mercado (G-A) asume funciones que anteriormente estaban desempeñadas por otros dos sectores (I-L). Con la postmodernidad, estas últimas esferas de relaciones sociales no sólo no son superfluas, sino que se desarrollan de una forma imprevista. Los sistemas de protección social asumen que, hasta ahora, han basado sus actuaciones en el eje A-G, y que solamente han recurrido a L y a I para objetivos de suplencia e integración «subordinados» y "subsidiarios» al Estado.

En la sociedad postmoderna ya no es posible aceptar que el complejo I-L (en el que se expresa la sociedad civil) es el producto del complejo G-A (Estado-Mercado). Es necesario repensar el bienestar como producto de las interrelaciones entre A, G, I, L, es decir, a la luz de un sistema global de acción suficientemente diferenciado e integrado interna y externamente.

Si la idea de welfare mix no tiene un sentido banal, con ella se quiere indicar que el bienestar es el producto de un sistema generativo constituido por las relaciones entre las cuatro esferas fundamentales de la sociedad, y entre los cuatro tipos de actores, allí donde tales relaciones están gobernadas por tres grandes procesos sociológicos: diferenciación, reintegración y emergencia de intercambios interactivos. Veámoslos más en detalle.

Entre los cuatro sectores existe un proceso de diferenciación societaria: los actores se diferencian continuamente entre sectores y en el interior de éstos:

A) Los actores de mercado (for profit) buscan nuevos paradigmas de utilidad interiormente y por diferenciación con el bienestar perseguido mediante acciones primariamente no utilitarias.

G) Los actores del sistema político-administrativo se diversifican a partir del crecimiento de complejidad de los aparatos que deben realizar la ciudadanía estatal.

I) Los actores de las esferas de solidaridad social non-profit (llamados de privado social, tercer sector, tercer sistema) se convierten en protagonistas de profundas diferenciaciones internas (voluntariado, cooperación social, asociaciones de cualquier tipo, fundaciones, trusts, etc.) y nuevas formas de intercambio con los otros sectores (por ejemplo, las bancas éticas, fondos de alimentos o farmacéuticos, etc.).

L) Las familias y redes informales redefinen el bienestar a lo largo de líneas de "familiaridad» que tienen sus propios medios específicos, finalidades, reglas y valores.

Existe la necesidad de un proceso de reintegración societaria: los diversos actores buscan lógicas combinatorias (entre códigos culturales y formas organi- 
zativas de protección social) que, si se utiliza el esquema AGIL, pueden presentar las siguientes tipologías:

- A-G: acuerdos neocorporativos.

- G-L: delegación de seguridad social del Estado a instituciones de privado social (véanse las leyes aprobadas en casi todos los Estados europeos en la última década para valorar las funciones del voluntariado y de la cooperación social al servicio de las instituciones del sistema político-administrativo).

- I-L: sujetos de tercer sector, desde los más organizados (empresas sociales, voluntariado, cooperación social) a los más informales (mutualidad, self-help, etc., con la acción directa de las familias).

- A-I: acuerdos entre mercado for profit y economía social non for profit.

- G-L: transferencias directas del Estado a las familias y, viceversa, organismos de representación de las familias para su protección hacia el Estado (por ejemplo: consejos de los usuarios o de los consumidores).

- A-L: acuerdos entre hacienda y familia (por ejemplo: contratos para la sustitución del padre por el hijo en el puesto de trabajo).

- A-G-I: esquemas de protección social gestionados por el mercado, el Estado y el tercer sector.

- G-A-L: esquemas de protección social gestionados por el Estado, el mercado y las familias.

- G-I-L: esquemas de protección social gestionados por el Estado, el tercer sector y las familias.

- A-I-L: esquemas de protección social gestionados por el mercado, tercer sector y las familias.

- A-G-L-I: programas de desarrollo de comunidad (community development) como modelo de política social para un reagrupamiento social o territorial, en cuyo interior se ubican las asistencias de comunidad (community care) como intervención de sector en el reparto socioasistencial; un fenómeno reciente, interno a esta especie de programas, es el de las "fundaciones de comunidad»". De la necesidad de estas combinaciones nacen nuevas formas de intercambio.

Mediante diferenciaciones y reintegraciones, emergen nuevas configuraciones de los procesos de intercambio entre los cuatro ámbitos mencionados, sus sujetos y los códigos culturales: es necesario ver que se intercambian entre sí (input y output), cómo lo hacen y qué emerge. Tendremos que analizar quépueden contener y representar las flechas y los números de la figura 1 en términos de acciones y aportaciones para el bienestar de los individuos, las familias y los grupos sociales (bienes producidos en cualquier intercambio).

1. ¿Qué da el sistema político-administrativo al mercado? Regulación para garantizar y tutelar, pero también un sistema de incentivos y sanciones

9 Véase B. Casadei (1997). 
que orientan a los actores de mercado hacia modalidades de producir, distribuir y consumir bienestar. También hay que tener en cuenta que estas modalidades de regulación política se reflejan indirectamente sobre los intercambios que, a su vez, el mercado tiene con el resto de esferas.

2. ¿Qué da el mercado al sistema político-administrativo? Recursos financieros bajo la forma de contribuciones fiscales. Pero también input para la definición de los standards de consumo de bienes y servicios de bienestar.

3. ¿Qué da el sistema político-administrativo al tercer sector? Las mismas prestaciones que da al mercado, es decir, regulación para garantizar-tutelar y un sistema de incentivos-sanciones. ¿Cuál es la diferencia? Debería ser un trato diferencial entre organizaciones de benéfico y no de beneficio (profit/non-profit), pero no siempre es así. Por otra parte, el mejor trato fiscal hacia el non profit generalmente contiene mayores vínculos que para las agencias de beneficio.

4. ¿Qué da el tercer sector al sistema político-administrativo? Sustitución y apoyo para funciones que los aparatos estatales no están en situación de ofrecer. Especialmente en la gestión de las capas más débiles y marginales de la población.

5. ¿Qué da el sistema político-administrativo a las familias y a las redes informales? Lo mismo que da al mercado y al tercer sector. Pero aquí se plantea el problema: ¿con qué diferencias de trato y resultados?

6. ¿Qué da el sistema de las familias y de las redes informales al sistema político-administrativo? Legitimación política más o menos consensuada que se concreta mediante peticiones, pero también ayudas. Las familias y el Estado son subsidiarios entre sí.

7. ¿Qué da el tercer sector al sistema de las familias y de las redes informales? La primera prestación funcional es la organizativa y managerial, es decir, la posibilidad para las familias y relaciones informales de encontrar ámbitos organizativos de un nivel más elevado.

8. ¿Qué da el sistema de las familias y de las redes informales al tercer sector? En primer lugar, motivaciones psicológicas y culturales para sostener la acción, pero también ayudas concretas.

9. ¿Qué da el mercado al sistema de las familias y de las redes informales? Bienestar privado mediante pago, y con ello incentivos y sanciones para particulares estilos de vida.

10. ¿Qué da el sistema de las familias y de las redes informales al mercado? Peticiones de prestaciones de bienestar y orientaciones al consumo.

11. ¿Qué da el tercer sector al mercado? Competencia en el sector de la solidaridad social.

12. ¿Qué da el mercado al tercer sector? Generalmente apoyos de bienestar en términos de beneficencia, pero también estímulos para capacidad empresarial diversa de la capitalista (bancas éticas, participación de empresas for profit en actividades non profit, etc.).

El análisis es muy complejo. Se debería realizar no sólo en la concreta dirección (one way) de una esfera a otra, también en el producto que emerge 
(fenómeno emergente) de todo intercambio y en los resultados que los intercambios directos e indirectos tienen en otras esferas y sobre otras relaciones entre esferas. En estas páginas basta con sugerir el cuadro conceptual y la metodología para tal análisis, que debería ser contextualizada para cualquier situación y/o medida de bienestar. En cualquier caso, este esquema revela que el welfare mix asume un carácter sui generis.

El welfare mix, en su forma más completa y compleja, está constituido por el diagrama de flujo combinatorio y relacional de la figura 1, que visualiza la actual morfogénesis del bienestar:

- Desde la diferenciación, el welfare mix significa: pluralidad de actores y pluralidad de códigos culturales del bienestar.

- Desde la integración entre actores y códigos culturales diferenciados, el welfare mix significa: combinación entre sí y reticularidad de sus formas de actuar y cooperar.

- Desde la emergencia de nuevos bienes y servicios, el welfare mix significa: mayor distinción entre bienes-servicios privados, públicos y mixtos. Por tanto, la posibilidad de que afloren nuevas modalidades de entender y practicar el bienestar también como vida buena. De esta forma, el bienestar se redefine continuamente en sus componentes fundamentales (medios económicos, objetivos, normas, referencias a los valores), según esquemas relacionales. Todo ello permite producir nuevos bienes como aquellos cuyo contenido no es una mera prestación funcional, sino las mismas relaciones sociales (en las que los operadores intervienen). A estos bienes los denominaremos «bienes relacionales» ${ }^{10}$.

Obviamente, las tres dinámicas que caracterizan el welfare mix (es decir: diferenciación, integración y emergencia de nuevos bienes y servicios, mediante la redefinición de los intercambios entre dimensiones y sujetos de bienestar) pueden tener lugar de otras formas muy diversas. Detrás de estos procesos de diferenciación, articulada e integrada, de los diversos subsistemas y funciones de la sociedad, no sólo existe crisis y fragmentación ${ }^{11}$. También algunas nuevas líneas de un diseño más complejo de protección social. Como tal, también es más contingente, cargado de paradojas y ambivalencias, poco transparente en cuanto a sus lógicas explícitas y directas. ¿Hay un código que la gobierna? O bien: ¿a partir de qué lógicas tiene lugar todo? Es cuanto analizaremos en el siguiente apartado.

\section{CÓMO CAMBIA EL SENTIDO DE LA POLÍTICA SOCIAL}

El welfare mix puede o no introducir el debate sobre el Estado de Bienestar tradicional. La lógica de la complejidad relacional que se ha descrito (fig. 1)

${ }^{10}$ Véase P. Donati (1993: cap. 2).

11 Véase AA.VV. (1996). 
puede ser gestionada según las tendencias de la modernidad, o bien según un nuevo espíritu postmoderno de las combinaciones y de las relaciones que da vida a una política social diferente de la típica de la ciudadanía moderna. En resumen, existen dos formas de ver y activar el welfare mix: como simple adaptación o como cambio, más o menos radical, de paradigma.

A continuación examinaremos estos dos modelos de entender y practicar las políticas sociales que, por simplicidad, llamaremos modelo lib/lab y modelo societario.

En el modelo lib/lab, el welfare mix se gestiona de la siguiente forma:

- El eje principal sigue siendo el binomio Estado-Mercado, confiando al primero la regulación (titulaciones de ciudadanía social) y al segundo la desregulación (libertad).

- El tercer sector asume un rol de apoyo al binomio Estado-Mercado como sujeto de integración.

- A las familias y las redes informales se las trata como sujetos débiles que tienen que ser asistidos con medidas de bienestar garantizado por «terceras partes» (respecto a las transacciones entre sí o con el puro mercado).

Las palabras clave de este modelo son inclusión y cohesión social, o bien cohesión social mediante la progresiva inclusión de las capas de población en el sistema lib/lab. El welfare mix es utilizado, por así decir, como instrumento más flexible y sofisticado para una inclusión política, aun concebida según los cánones de la modernidad.

Sin embargo, en el modelo societario, el welfare mix se gestiona así:

- En el binomio Estado-Mercado se introduce una concepción subsidiaria en sentido vertical y horizontal de las instituciones de bienestar que: traslada a un complejo de ciudadanía donde adquieren vigor derechos civiles, políticos, sociales y humanos, confiando a cualquier sujeto la regulación de la relación entre libertad y responsabilidad, entre costes y beneficios, en el ámbito de una división funcional del poder (que mantiene en el sistema político-administrativo las decisiones que, en la esfera común, son vinculantes para todos).

- El tercer sector asume un rol institucional autónomo, simétrico respecto al Estado y el Mercado, como sujeto alternativo en la promoción del bienestar.

- Las familias y las redes informales son promovidas como sujetos activos que producen, distribuyen y redistribuyen un bienestar reconocido como autónomo (aunque no separado) del sistema político-administrativo.

Las palabras clave de este modelo son: pluralismo de las lógicas reticulares y de la promoción de autonomías sociales capaces de producir inclusión y cohesión social, no mediante medidas uniformes y derechos abstractos de ciudadanía, sino a través de subjetividades sociales en las que se expresa el welfare mix. Dicho en otros términos, el welfare mix no es considerado en sentido ins- 
trumental, sino como vía de revitalización y promoción de formaciones sociales poseedoras de derechos-deberes diferentes de los correspondientes a los individuos o a la colectividad política en su conjunto. El mix no es contemplado como simple adaptación organizativa para la actuación de la ciudadanía estatal. Se convierte en un sistema morfogenético que valora las formas asociativas que realizan la inclusión política mediante el principio de ciudadanía societaria, concebida en términos postmodernos como expresión de la sociedad civil antes que como pertenencia a un Estado-nación.

Puede ser útil comparar estos dos modelos mediante algunas dimensiones fundamentales de su configuración (fig. 2) que, por razones de espacio, no comentaremos.

\section{FIGURA 2}

\section{Dos formas de entender y practicar las políticas sociales en el welfare mix}

\begin{tabular}{|c|c|c|}
\hline Caracteristicas & Lógicas lib/lab & Lógicas societarias \\
\hline $\begin{array}{l}\text { Principio de ciu- } \\
\text { dadanía. }\end{array}$ & $\begin{array}{l}\text { Maximización de la inclusión polí- } \\
\text { tica igual para todos. }\end{array}$ & $\begin{array}{l}\text { Generalización de garantías mínimas } \\
\text { y diferenciación del bienestar según } \\
\text { las pertenencias socioculturales. }\end{array}$ \\
\hline $\begin{array}{l}\text { Referentes de las } \\
\text { políticas sociales. }\end{array}$ & $\begin{array}{l}\text { Categorías sociales definidas en pri- } \\
\text { mer lugar por relación al mercado } \\
\text { capitalista. }\end{array}$ & $\begin{array}{l}\text { Condiciones y estilos de vida de } \\
\text { toda persona en las diversas forma- } \\
\text { ciones sociales. }\end{array}$ \\
\hline $\begin{array}{l}\text { Cobertura de los } \\
\text { riesgos. }\end{array}$ & $\begin{array}{l}\text { Aseguraciones obligatorias públicas } \\
\text { y privadas para categorías profesio- } \\
\text { nales y compensaciones a las capas } \\
\text { marginales por vía fiscal. }\end{array}$ & $\begin{array}{l}\text { Nuevos mix de cobertura de los } \\
\text { riesgos mediante combinaciones } \\
\text { entre aseguraciones obligatorias, } \\
\text { integrativas, formas de mutualidad } \\
\text { y de solidaridad primaria. }\end{array}$ \\
\hline
\end{tabular}

Reglas de coloca- Prevalencia de reglas distributivas ción. con integraciones de contratación libre.

Rol del Estado.

Estado social institucional con integraciones de bienestar adquisitivo-meritocrático.

Extensión de la ciu- Ciudadanía estatal y local extendidadanía. da a los que la merecen (nativos e inmigrantes o denizens).
Mix de reglas basadas en criterios de redistribución, cambio (reciprocidad) y distribución solidaria (mutualidad).

Estado subsidiario a las autonomías sociales tanto en sentido vertical como horizontal.

Ciudadanía plural, ya sea sobre bases territoriales, ya sea sobre bases de pertenencia social en las diversas formaciones sociales.

Personas "contextualizadas" (en "comunidades de identidades») y formaciones sociales intermedias entre individuo y Estado. 
Los dos modelos, o lógicas, que hemos individuado jamás se han realizado de una forma pura. En la literatura existente al respecto, la mayor parte de las posiciones teóricas y prácticas son posiciones intermedias entre los paradigmas apuntados (fig. 2). Veamos algunas.

N. Johnson habla de un "pluralismo de welfare» como mera pluralización de las agencias que elaboran servicios y prestaciones de bienestar ante la crisis de transición del Estado de Bienestar ${ }^{12}$. Por su parte, O. De Leonardis plantea un welfare mix como producto de la erosión entre los límites entre público y privado $^{13}$. P. Hirst apunta el tránsito "del estatalismo al pluralismo», allí donde el nacimiento de una pluralidad de sujetos de bienestar es contemplado en el cuadro de su financiación pública ${ }^{14}$; con ello, este autor revela una línea muy difundida que considera el welfare mix como "tercera via» entre capitalismo y socialismo utópico, una línea que renueva las mejores instancias de la socialdemocracia y que, por tanto, está más próxima a las posiciones lib/lab que a las societarias. En Europa, esta posición es defendida por aquellos autores que valoran el privado social como subsidiario del Estado, antes que como sujeto autónomo y alternativo a los servicios estatales. En su opinión, la lógica de las acciones del tercer sector - en cuanto depende de las pertenencias culturales e ideológicas - es particular y discrecional, susceptible de crear nuevas desigualdades y nuevas formas de exclusión. Por este motivo abogan por un welfare mix en el que el Estado conserve el poder de programación y de financiación de las políticas sociales, confiando a las organizaciones non profit sólo la gestión directa de los servicios ${ }^{15}$. Ignoran que un modelo de welfare mix que concede al privado social libertades limitadas y circunscritas a la gestión dirigida desde el Estado, incurre en notables efectos perversos de colonización de la sociedad civil. Aún más, dicho modelo devalúa a las agencias de solidaridad del sector non profit ${ }^{16}$.

La mayor parte de las posiciones citadas captan tendencias como la crisis del modelo de Estado de Bienestar total y la emergencia de nuevas formas de gestionar las relaciones entre libertades privadas y controles públicos ${ }^{17}$. Pero están a mitad de camino entre el paradigma lib/lab y el paradigma societario.

Por ejemplo, se habla de "casi mercados» ${ }^{18}$ para referirse a la separación entre consumidores y productores de servicios, y a la multiplicación (pluralización) de los unos y de los otros. Ciertamente, esto modifica la antigua configuración en la que actuaba un solo actor (el viejo Estado de Bienestar); pero está claro que no estamos ante verdaderos y propios mercados en cuanto que los servicios de bienestar se encuentran financiados y controlados por el sistema

12 Véase N. Johnson (1987).

13 Véase O. De Leonardis (1996).

${ }^{14}$ Véase P. Hirst (1997).

15 Véase C. Ranci (1999).

16 Véase L. Fazzi (1998).

17 Véase L. Mead (1986).

${ }^{18}$ Véase G. Wistow y J. Forder (1995). 
político-admistrativo (entes públicos centrales o locales). Por otra parte, se observa que si los llamados casi-mercados disminuyen los costes de producción, elevan los costes de transacción, de tal manera que las ventajas netas finales en términos de racionalización económica-son bastante escasas.

Las corrientes dominantes —incluso en las políticas de la Unión Europea- buscan nuevas combinaciones entre el máximo de libertad y un orden social que socialice el mínimo indispensable de bienestar. Razonan en términos de compatibilidad y de bienestar sostenible, antes que adoptar un nuevo modelo generativo. En este sentido, se encuentran más próximas también al modelo lib/lab que al societario.

Pasar a este último paradigma presupone una visión relacional de la sociedad. Visión que sea capaz de trascender la dialéctica típicamente moderna entre libertad (lib) e igualdad (lab). Y de esta forma dar mayor vigor al polo de las autónomas solidaridades sociales que producen, distribuyen y consumen bienestar, siendo tal polo ubicado en un status simétrico respecto al Estado y el Mercado, con capacidad propia de combinación de los recursos en un contexto de globalización ${ }^{19}$.

Lo que está en juego es el sentido político del trabajo social en una nueva formación histórico-social. Los fenómenos estructurales y culturales de largo alcance que trasladan al cambio del sentido político del trabajo social son:

- La erosión de las líneas de división entre público y privado en las políticas de bienestar.

- La caída de los significados y de las prácticas de libertad y de control social.

- La caída de los nexos entre riesgos y responsabilidades personales y colectivas.

- La mutación de las formas de vida social, con relaciones sociales más contingentes y sujetas a dinámicas de distribución-creación continua, y el surgimiento de nuevos estilos de existencia en los que el bienestar social es muy problemático.

En el fondo, el debate se centra en el sentido del trabajo social en cuanto que ya no puede ser gobernado desde el sistema político-administrativo. Más bien necesita una sociedad civil que, al mismo tiempo, se presenta más dinámica, pero también más caótica, incontrolable, ambivalente, paradójica y, por tanto, fuente de nuevos recursos y de nuevos riesgos. El trabajo social está marcado por la irrupción de esta nueva realidad que denominamos con el viejo término de sociedad civil, aunque no sabemos por qué las categorías hasta ahora utilizadas no corresponden a cuanto expresa.

El nudo del welfare mix está ahí: en la relación, siempre más compleja y problemática, entre el sistema político-administrativo y la sociedad civil,

19 Véase J. Midgley (1997). 
teniendo en cuenta que las categorías tradicionales con que la concebimos no son de mucha ayuda. No es posible pensarla en términos de los modelos liberales versus comunitarios, con los que contraponer el clásico paradigma tocquevilliano de una sociedad civil entendida como democracia fuerte basada en una cultura de civismo republicano ${ }^{20}$. Todo este conjunto de categorías conceptuales (y prácticas) actualmente es obsoleto ${ }^{21}$. Para saber dónde va y/o pueda ir el trabajo social es necesario comprender cómo se modifica su significado «politico» ante las nuevas instancias civiles.

\section{LA POSICIÓN «POLÍTICA» DEL TRABAJO SOCIAL: ¿AÚN SON «POLÍTICAS» LAS POLÍTICAS SOCIALES?}

En el Estado de Bienestar clásico, la posición política del trabajo social era muy clara. Cualesquiera que fuesen las intenciones o las manifestaciones declaradas o escondidas, explícitas o implícitas, se trataba de una acción de emancipación realizada por la comunidad política —organizada bajo la forma de Estado- en sus relaciones con las clases sociales no privilegiadas. En la actualidad, esa ética del asistente social sigue estando presente en el interior de ese marco político. Se trataba de una posición que no era cómoda. En efecto, la posición política del trabajador social era la de un double bind. Los trabajadores sociales recibían dos mensajes opuestos entre sí: por una parte, debían ayudar a los más desaventajados y representar sus intereses; por otra, eran ellos mismos los privilegiados (por tener una posición de mando y un trabajo profesional estable), defendiendo un sistema que generaba pobreza, marginación y exclusión. R. Titmuss lo había captado perfectamente cuando llamaba a los trabajadores sociales «los trabajadores del Estado». No en vano debían defender un orden social basado en los privilegios que generaban los problemas sociales que debían resolver ${ }^{22}$.

Esta posición estructural intrínsecamente contradictoria constituye el origen de una serie de debates. Éstos han revelado el rol político esquizofrénico y ambiguo del trabajador social dentro del Estado de Bienestar moderno, de tipo panóptico y posteriormente fordista. En él, las políticas sociales eran, en buena medida, políticas de colonización de los mundos vitales ${ }^{23}$.

Con la crisis del Estado de Bienestar, su desestructuración y pluralización, y posteriormente con el nacimiento del welfare mix, la posición estructural del trabajo social se modifica radicalmente:

- El sistema de bienestar no tiene ya un «vértice» (un poder superior de referencia para todos).

${ }^{20}$ Como hace B. R. Barber (1998).

${ }^{21}$ Véase P. Donati (1997).

${ }^{22}$ Véase R. Titmuss (1978).

${ }^{23}$ Véase P. Donati (1981). 
- El sistema de bienestar ya no tiene un «centro», ni de coordinación ni de dirección.

- El sistema de bienestar ya no tiene sólo una o prevalente imputación de responsabilidad colectiva.

- El sistema de bienestar se expande uniformemente en todas las direcciones. El resultado es que cualquier punto se distancia de los otros, pero también se encuentra ligado al movimiento general que afecta a todos los puntos en los que el bienestar se produce y consume.

- El bienestar se hace "autopoiético», es decir, debe producirse en el interior de cualquier sistema organizativo según su propia distinción-directriz.

Emerge un sistema de protección social en el que el trabajo social adquiere connotaciones inéditas. El rol del operador social se caracteriza por:

- Ser un rol de partnership, de combinación entre varios roles, pertenecientes tanto a los aparatos estatales, a las asociaciones voluntarias, como a las redes informales.

- Como actividad de redes que necesita de un alto nivel de movilidad y flexibilidad.

- Como circularidad de las competencias.

- Con responsabilidades progresivamente más «locales».

- Adaptable según nuevas formas de contratación.

Cuando se declinan desde una perspectiva plenamente relacional, tanto el concepto de community care ${ }^{24}$ como el de "trabajo de redes» ${ }^{25}$ captan muchas de estas novedades ${ }^{26}$. Detrás de todo ello se esconde un código simbólico de la red como "metáfora de pertenencia» ${ }^{27}$. En tal contexto, los trabajadores sociales tienen muchas y diversas posibilidades de escapar al double bind. Pero también son mayores los vínculos y los riesgos. Buena muestra de ello es que la ética del trabajo social, más allá de la persistencia de referentes tradicionales, debe afrontar nuevos dilemas y termina por orientarse hacia sistemas de referencia — llamados postmodernos — fuertemente desestructurados. En ellos los códigos éticos de los órdenes profesionales son desafiados por una creciente subjetividad y fragmentación ${ }^{28}$.

Nos podemos preguntar cómo los modelos de welfare mix (lib/lab y societario) captan las diversas perspectivas del trabajo social que se abren en la época

${ }^{24}$ Véanse F. Folgheraiter y P. Donati (1991) y M. Herrera (1998a).

25 Véanse L. Sanicola (1997) y F. Folgheraiter (1998).

${ }^{26}$ Una visión relacional de la asistencia de comunidad, basada en la experiencia británica del National Health Service and Community Care Act del 1990, puede encontrarse en M. Barnes (1997).

27 Véase P. Di Nicola (1998).

28 Véase S. Banks (1995). 
postmoderna y, en consecuencia, cómo conciben la posición política del trabajo social.

El paradigma lib/lab no parece captar el sentido de las rupturas con el pasado que se presentan en el nuevo escenario. Insiste en las viejas líneas-guía de la política social, en concreto contra las discriminaciones, la exclusión social y la desigualdad de oportunidades ${ }^{29}$. Tales objetivos aún deben afrontarse. Pero la forma de perseguirlos ya no funciona. Se ha demostrado que una política social del tipo lib/lab pierde completamente el carácter político: la política lib/lab sólo puede definir los derechos sociales y humanos en negativo y no en positivo, no es útil para prevenir las patologías sociales, y procede más por exclusión que por inclusión ${ }^{30}$. Desde este camino, el trabajo social no puede evitar las tentaciones neopanópticas que, en ciertas áreas de Europa, están sustituyendo la política social con la política penal ${ }^{31}$.

El modelo que hemos llamado societario ofrece un mayor respeto tanto a la autonomía del sistema de bienestar en su conjunto respecto al resto de sistemas y formas organizativas de la sociedad, como a la del trabajador social en las diversas dimensiones del bienestar (culturales, manageriales, etc.). Sin embargo, el escenario de una ciudadanía societaria y de sus respectivas políticas sociales no se encuentra determinado. Por una parte, es cierto que el modelo lib/lab intenta desesperadamente mantener el carácter político de las políticas sociales como responsabilidad colectiva del Estado, o de entidades funcionalmente equivalentes, encontrando crecientes fracasos. Por otra, muchos lamentan que el modelo societario también pierda el carácter político del trabajo social y de las correspondientes políticas sociales. Quien sostiene esta tesis ve en el modelo societario fragmentación, así como un terreno fértil para procesos capitalistas aún más competitivos, agresivos y desigualitarios. El interrogante a afrontar es el siguiente: en el caso del modelo societario, ¿las políticas sociales son aún políticas?

Ciertamente lo son, pero en un sentido totalmente nuevo. El carácter político ya no es el de un sistema (Estado-nación) que dirige y eventualmente se descentraliza. Pero es por su naturaleza descentralizada en todo sistema operativo, donde está presente como proceso decisiones que debe conducir a un bien común no general-genérico sino contextualizado. El universal se deferencia, incorpora (embodded) e introduce (embedded) en el particular. A su vez, el particular debe ser expresión de valores universales. Las unidades superiores deben ser concebidas como asociaciones de tales sistemas operativos (que son asociaciones). Son las asociaciones de asociaciones quienes deben realizar el trade off entre universal y particular.

La vía societaria modifica profundamente el sentido político de las nuevas políticas sociales. En esta línea: a) lo político, entendido como producción de

29 Véase, por ejemplo, el Forum europeo 1998 de las políticas sociales, EU (1999).

${ }^{30}$ Véase N. Luhmann (1983 y 1995).

${ }^{31}$ Véase AA.VV. (1998). 
un bien común, se diferencia de la política, entendida como actividad inherente al sistema político-administrativo ${ }^{32}$; y $b$ ) lo político, antes que erosionarse, es situado en cualquier unidad operativa, y es controlado mediante dinámicas de "asociaciones que hacen asociación». Aquí es posible un trabajo social proyectivo, entendido como guía relacional y como desarrollo de los derechos sociales y humanos ${ }^{33}$ mediante una nueva sociedad civil hecha de sentido político como sentido asociativo ${ }^{34}$.

\section{CONCLUSIONES: LOS PRINCIPIOS-GUÍA DEL WELFARE MIX}

En Europa, los sistemas de protección social han entrado en crisis con el tránsito de la sociedad industrial a la postindustrial. Aún es un problema comprender en qué consiste este proceso "societario», proceso que comporta una redefinición de la seguridad social en el marco de un sistema de protección social más amplio.

Las dificultades para acceder a nuevos sistemas de protección social están ligadas al siguiente hecho: prevalecen las principales instituciones (y sus correspondientes mecanismos) de seguridad social tipo industrial (es decir, una seguridad social diseñada sobre relaciones industriales y sobre problemas del mercado de trabajo); mientras que la seguridad social sólo puede funcionar bien si cuenta con un sistema más amplio, articulado e integrado de intervenciones de protección social. Dicho en otros términos, con una concepción global del welfare mix.

Actualmente dominan las tendencias de la neta separación entre seguridad y asistencia social. Dichas tendencias provocan notables dificultades para grupos sociales de marginados y de excluidos del sistema de protección social. Allí donde falta un amplio sistema de protección social existe un retroceso antes que la construcción de instituciones de bienestar adecuadas a los riesgos de una sociedad compleja. Se puede intentar incluirlos por vía política, según el principio moderno de la ciudadanía total. Pero esto ya no es posible. Lo cierto es que el welfare mix cambia el sentido de lo que, en políticas sociales, es y puede ser "politico».

El modelo más desarrollado de seguridad social —aquel institucionalizado en los regímenes democráticos de bienestar neocorporativo-, al mismo tiempo que no consigue resolver los problemas tradicionales (especialmente la desocupación y la pobreza), no afronta los nuevos desafíos constituidos por nuevos riesgos y nuevos procesos de marginación social. Estos desafíos se manifiestan en cambios radicales en: a) el ciclo de la vida de los individuos y

32 Véase A. Caillé (1993).

33 Véase P. Donati (1991: cap. 5).

34 Se habla de "ciudadanía profunda" (P. B. Clarke, 1996), de "democracia asociativa» (J. Cohen y J. Rogers, 1993; P. Hirst, 1994; E. O. Wright, ed., 1995), de «socioeconomía del hecho asociativo» (A. Caillé y J. L. Laville, 1998). 
de las familias; $b$ ) los estilos de la vida cotidiana; $c$ ) el número y problemas de las personas que no tienen ciudadanía (refugiados, inmigrantes, trabajadores temporales, nuevos nómadas, etc.), y d) los riesgos ambientales (una compleja «sociedad arriesgada») que requieren mayores controles tanto a escala macro como a escala local.

En este escenario es necesario un nuevo diseño de la protección social. El punto de discontinuidad viene dado por el cambio del sentido "político" en hacer el trabajo social y las políticas sociales. En Europa se intenta salir del viejo modelo intervencionista neocorporativo. Pero tanto el new labour de Toni Blair como el llamado «modelo holandés» ponen de manifiesto que todos estos intentos se enmarcan en el modelo lib/lab. Éste trata de buscar soluciones en el trade off entre más libertad de mercado y más regulación del Estado. Otros países como Italia o España, en los que aún domina el modelo precedente de neocorporativismo democrático, no consiguen caminar hacia las configuraciones lib/lab.

Como hemos señalado anteriormente, se debería revisar el cuadro lib/lab en el que actualmente se definen las políticas sociales, y se debería pensar en un nuevo diseño de bienestar que tendría que basarse en: $a$ ) la pluralización de los actores que producen y gestionan el bienestar; b) una mayor autonomía, inspirada en un cuadro de mayor diferenciación de los sistemas de protección social micro y macro; c) confiar a las instituciones del Estado Social un rol de «regulación» (ordenador general) y de "guía relacional» (promoción subsidiaria) encaminados a la emancipación de un bienestar civil.

Los países más retrasados (laggards) en la construcción de un Estado de Bienestar moderno, como Italia y España, han sido hasta finales de los años ochenta el emblema de un sistema de seguridad social entendido como complejo previsor-asegurador diseñado por una «sociedad industrial estatalizada» que el Estado nacional jamás ha conseguido controlar. Las medidas privatizadoras y de modernización de la década de los noventa son insuficientes para afrontar la gravedad de los desafíos. Las reformas de los sistemas de protección social actualmente intentan moverse en una dirección más innovadora. Pero lo hacen desde el interior de viejos esquemas, es decir, según modalidades que tienen, por una parte, como legitimación ideológica el modelo lib/lab y, por otra, como prácticas efectivas algo que se asemeja a las lógicas societarias, pero distorsionadas por el cuadro normativo lib/lab.

En una situación de creciente postmodernidad, la protección social tiene necesidad de individuar nuevos actores, nuevos principios-guia y nuevas reglas. Más allá de la vinculación con el Estado (o sistema político-administrativo) en términos de armonización, convergencia y coordinación, deberán ser capaces de autodirección y autocontrol en términos de eficacia, eficiencia y equidad en los costes y en las ventajas de la protección social colectiva. El problema consiste en regular la mayor autonomía de las formas de protección social autogestionadas de los diversos actores interesados en ella con la equidad social (entre categorías, clases y generaciones diversas). 
Dicho en otros términos, es necesario que los sistemas de protección social consigan encontrar un nuevo equilibrio entre las formas de "integración sistémica» (organizaciones formales) y las formas de "integración social» (asociacionismo social difundido en "redes» a escala de comunidad y en las relaciones formales/informales), dentro de una nueva configuración relacional de "ciudadanía societaria».

Los principios que pueden ayudar a alcanzar estos objetivos son los siguientes:

- Subsidiariedad vertical (dentro del sistema político-administrativo territorial) y horizontal (entre el sistema político-administrativo y la sociedad civil).

- Regulación sobre principios universales.

- Autonomía de las organizaciones operativas, con controles (de eficiencia, eficacia, equidad y calidad) en sus outputs concretos (antes que ex ante sobre sus estructuras internas).

El carácter político de las políticas sociales ya no se deriva del hecho de ser una emanación del Estado (o del sistema político-administrativo) con sus funciones de dirección en los diversos niveles territoriales. Más bien emerge un nuevo modo de percibir y hacer lo político, interno a las diversas esferas sociales que emanan de la sociedad civil. En este sentido, la actividad del trabajador social puede ser más política si se activa una reflexividad suficiente. Ésta debe ubicar al trabajador en situación de comprender que se trata de una política societaria y no estatal, en cuanto que el carácter político ya no se refiere al Estado, ni siquiera a la antigua polis, sino al sistema asociativo de las organizaciones que producen-distribuyen-consumen bienestar.

Concretamente, en los países mediterráneos el servicio social aún presenta notables resistencias a los cambios. Persisten los roles tradicionales (dependientes y ejecutivos), y se ponen de manifiesto dinámicas de profesionalización de los servicios en términos de una racionalización que concede poco a lo civil, aunque se introduce alguna innovación por imitación de experiencias externas, en concreto anglosajonas. Por ejemplo, la promoción de la figura del case manager $^{35}$, que aún no posee las connotaciones de autónoma profesionalidad que debería caracterizarla para que no sea absorbida por la lógica burocrática típica del funcionariado ${ }^{36}$.

35 Véase M. Payne (1995).

${ }^{36}$ Debe señalarse que, también externamente, la figura del case manager se ha burocratizado a medida que ha sido absorbida por la configuración lib/lab, antes que convertirse en una figura societaria. M. Payne (1999) distingue tes tipos: el empresarial, el de intermediación (brokerage) y el de keyworker en equipos multiprofesionales. El mismo autor ha admitido que cuando el case manager pierde autonomía y es absorbido por el sistema público (estatal, bien central o local) de control, los fracasos son más frecuentes (muy buen ejemplo es la experiencia inglesa). Todo ello confirma nuestro diagnóstico: el mayor carácter promocional que posee la configuración societaria frente al modelo lib/lab, que se centra en el juego libertad/control. 
En cualquier caso, está abierto el camino para hacer del trabajo social una acción que promueva un nuevo sentido político de las políticas sociales, como producción de bienes relacionales en una economía civil de los servicios. El desarrollo de estas formas depende de varias condiciones. En primer lugar, de que se pueda abandonar el estilo sindical-reivindicativo que el servicio social ha asumido en la fase histórica del Estado de Bienestar neocorporativo. En otros términos, el servicio social aún debe comprender qué significa poder ser protagonista si y en el momento en que asume el rol de promoción de las autonomías sociales de welfare mix.

El horizonte de tales posibilidades está marcado por una community care basada en un mix de redes formales e informales que actualizan organizaciones flexibles y con conexiones entre libertad y control, entre responsabilidades individuales y colectivas, entre riesgos y contribuciones. Indudablemente, estamos ante un nuevo escenario. Nuevo para quien trabaja en los entes públicos (estatales o dependientes del Estado), donde aún las prácticas basadas en metodologías relacionales son escasas o ausentes, mientras están emergiendo en el trabajo social orientado por el tercer y cuarto sector. Es decir, en el mundo del privado social organizado (cooperación social, voluntariado organizado, asociacionismo) y en las redes informales (de mutua y autoayuda) que se interseccionan con saberes expertos, dando vida a prácticas de welfare mix societario antes que lib/lab.

\section{BIBLIOGRAFÍA}

AA.VV. (1996), "Welfare mix. Stato, mercato, terzo settore: luci e ombre di uno scenario mobile», número único L'Assistenza Sociale, n. ${ }^{\circ} 1$, enero-marzo.

- (1998), "De l'État social à l'état pénal», número monográfico Actes de la recherche en scinces sociales, n. ${ }^{\circ} 124$, septiembre.

BANKS, S. (1995), Ethics and Values in Social Work, London, Macmillan (tr. it.: Etica e valori nel servizio sociale. Dilemmi morali e operatori riflessivi nel welfare mix, Trento, Erickson, 1999).

Barber, B. R. (1998), A Place for Us. How to Make Society Civil and Democracy Strong, New York, Hill and Wang.

Barnes, M. (1997), Care, Communities and Citizens, London-New York, Longman (tr. it.: Uten$t i$, carer e cittadinanza attiva. Politiche sociali oltre il welfare state, Trento, Erickson, 1999).

Barry, N. (1990), Welfare, Milton Keynes, Open University Press.

Bulmer, M. (ed.) (1989), The Goals of Social Policy, London, Unwin Hyman.

Caillé, A. (1993), La démission des clercs. La crise des sciences sociales et l'oubli du politique, Paris, La Découverte (tr. it.: Il tramonto del politico. Crisi, rinuncia e riscatto delle scienze sociali, Bari, Edizioni Dedalo, 1996).

Caillé, A., y Laville, J.-L. (eds.) (1998), «Une seule solution, l'association? Socio-économie du fait associatif», Revue du MAUSS, n. ${ }^{\circ} 11$, primer semestre.

CASADEI, B. (1997), «Le community foundations: una scelta strategica per le fondazioni delle casse di risparmio", en AA.VV., Fondazioni e organizzazioni non profit in Usa. Percorsi possibili per la realtà italiana, Rimini, Maggioli, pp. 171-186.

Cohen, J., y Rogers, J. (1993), Associative Democracy, en P. Bardhan y J. Roemer (eds.), Market Socialism, New York, Oxford University Press. 
Clarke, P. B. (1996), Deep Citizenship, London-Chicago, Pluto Press.

Colozzi, I. (ed.) (1987), La riforma dello Stato sociale. Un confronto europeo, Milano, Angeli.

De Leonardis, O. (1996), "Il welfare mix. Privatismo e sfera pubblica», en Stato e Mercato, n. ${ }^{\circ}$ 46, abril, pp. 51-75.

Di Nicola, P. (1998), La rete: metafora dell'appartenenza. Analisi strutturale e paradigma di rete, Milano, Angeli.

Donati, P. (1978), Publico e privato, fine de una alternativa?, Milano, Angeli.

- (1981), "L'operatore assistente sociale di fronte alla crisi del welfare state: quali prospettive?», en Studi di Sociologia, a. XIX, n. ${ }^{\circ}$ 1, pp. 3-22.

- (1991), Teoria relazionale della società, Milano, Angeli.

- (1993), La cittadinanza societaria, Roma-Bari, Laterza.

- (1997), «Alla ricerca di una società civile. Che cosa dobbiamo fare per aumentare le capacità di civilizzazione del Paese?», en P. Donati (ed.), La società civile in Italia, Milano, Mondadori, pp. 21-80.

- (1998), «Ripensare il welfare in Europa: oltre il lib/lab, verso un nuovo "complesso societario"”, en Sociologia e politiche sociali, a. 1, n. ${ }^{\circ} 1$.

- (1999a), "Il welfare del XXI ${ }^{\circ}$ secolo: perché e come dobbiamo inventarlo», en P. Donati (ed.), Lo Stato sociale in Italia: bilanci e prospettive, Milano, Mondadori.

- (1999b), «Famiglia e società del benessere: paradossi e contro-paradossi, mito e anti-mito», en P. Donati (ed.), Famiglia e benessere, Cinisello Balsamo, Edizioni S. Paolo.

Donati, P., y Colozzi, I. (1988), «Institutional Reorganization and New Shifts in the Welfare Mix in Italy During the 1980s», en A. Evers y H. Wintersberger (eds.), Shifts in the Welfare Mix, Vienna, Eurosocial, pp. 63-97.

EU (1999), «Dossier: European Social Policy Forum 98», en Employment \& Social Affairs, n.o 3, febrero.

FAZZI, L. (1998), Il welfare mix in Italia: primi passi, Milano, Angeli.

Folgheraiter, F. (1998), Teoria e metodologia del servizio sociale. La prospettiva di rete, Milano, Angeli.

Folgheraiter, F., y Donati, P. (eds.) (1991), Community care. Teoria e pratica del lavoro sociale di rete, Trento, Edizioni Centro Studi Erickson.

Herrera, M. (1998a), "Nuevas dimensiones en políticas sociales: la community care», en REIS, n. ${ }^{\circ} 82$, pp. $249-284$.

- (1998b), "Los sistemas de protección social en Europa», en Revista Internacional de Sociología, n. ${ }^{\circ}$, pp $16-52$.

Hirst, P. (1994), Associative Democracy. New Forms of Economic and Social Governance, Oxford, Polity Press.

- (1997), From statism to pluralism. Democracy, civil society and global politics, London, UCL Press (tr. it.: Dallo statalismo al pluralismo, Torino, Bollati Boringhieri, 1999).

IREF (1999), Combinare risorse. Strategie di co-finanziamento nelle organizzazioni di terzo settore italiane, Roma, Editoriale Aesse.

Jones, C. (ed.) (1993), New Perspectives on the Welfare State in Europe, London y New York, Routledge.

Johnson, N. (1987), The Welfare State in Transition. The Theory and Practice of Welfare Pluralism, Brighton, Wheatsheaf Books.

Luhmann, N. (1983), Teoria politica nello Stato del benessere, Milano, Angeli.

- (1995), «Das Paradox der Menschenrechte und drei Formen seiner Entfaltung e Inklusion/ Exklusion», en Soziologische Aufklärung 6. Die Soziologie und der Mensch, Opladen, Westdeutscher Verlag.

Marshall, T. H. (1950), Citizenship and Social Class, Cambridge, Cambridge University Press (trad. it.: Cittadinanza e classe sociale, Torino, Utet, 1976).

MEAD, L. (1986), Beyond Entitlement. The Social Obligations of Citizenship, New York, The Free Press.

Midgley, J. (1997), Social Welfare in Global Context, London, Sage. 
Montoro Romero, R. (1997), «La Reforma del Estado de Bienestar: Derechos, deberes e igualdad de oportunidades», en REIS, n. ${ }^{\circ}$ 79, pp. 9-42.

Payne M. (1995), Social Work and Community Care, London, Macmillan (tr. it.: Case management e servizio sociale. La costruzione dei piani assistenziali individualizzati nelle cure di comunità, Trento, Erickson, 1998).

- (1999), I "piani assistenziali individualizzati» nelle cure di comunità, intervención en el Congreso Internacional «Gli operatori sociali nel welfare mix», Università di Trento, Facoltà di Sociologia, D.U. en Servizio Sociale, Riva del Garda, 20-21 mayo.

RANCI, C. (1999), Oltre il welfare state. Terzo settore, nuove solidarietà e trasformazioni del welfare, Bologna, il Mulino.

SANICOLA, L. (1997), Itinerari nel servizio sociale, Napoli, Liguori.

TAVAZZa L., et al. (1998), Studies of - and Prospects for - the Revision of the Tax Laws Governing Non Profit Organizations, Roma, Fivol.

Titmuss, R. (1974), Social Policy: An Introduction, London, Allen \& Unwin.

Wilensky, H. (1976), The New Corporatism, Centralization and the Welfare State, London, Sage.

Wilensky, H. L., et al. (1985), Comparative Social Policy. Theories, Methods, Findings, Institute of International Studies, University of California.

Wistow, G.; Forder, J., et al. (1996), Social Care Markets: Progress and Prospect, Bukingham, Open University Press.

Wright, E. O. (ed.) (1995), Associations and Democracy, London-Nueva York, Verso.

\begin{abstract}
The handling of a sociological matter such as cultural «equipment» compels us to situate ourselves at a crossroads where subjects such as urban sociology, the sociology of culture or the sociology of daily life are thrown together.

The tackling of the causes of their genesis, as well as an approach to the way in which their logics of introduction occur, lead us to question some of the assumptions that are uppermost today and which, rather in the style of "preconceived notions», tend to establish themselves as an apparent explanation for specific policies and decision-making in this sphere.

Certain concepts such as "cultural democratisation", inspired in its origins by post-war progressive reforming policy in France, could have acted as a myth that obstructed discernment of the fact that policies producing cultural «equipment» have tended to become unified and independent of contextual decisions of a politico-ideological nature, retaining a strategic function as instruments in the service of reproduction of established local powers.
\end{abstract}

